Retinografía no midriática para la detección de la retinopatía diabética. Un ejemplo de adaptación al medio

\title{
Non-mydiatric retinography for the detection of diabetic retinopathy. An example of adaptation to the setting
}

\section{Forga}

Servicio de Endocrinología. Complejo Hospitalario de Navarra.
La retinopatía diabética $(\mathrm{RD})$ supone la mayor causa de ceguera en los países industrializados, por lo que implica una preocupación constante para los médicos que atendemos a los pacientes diabéticos. Su detección precoz, mediante la valoración del fondo de ojo, es fundamental para poder iniciar el tratamiento adecuado en el momento oportuno. La elevada prevalencia de la diabetes en la población general, fundamentalmente a expensas de la tipo 2, representa un problema para las consultas de Oftalmología que suelen ver una amenaza de colapso asistencial en esta patología. La búsqueda de posiciones intermedias, que permitan satisfacer la demanda sin bloquear la oferta, ha llevado al uso de la retinografía con cámara no midriática, aprovechando además la telemedicina para su valoración e informe clínico.

En Navarra disponemos de una experiencia pionera en este campo, que ha sido publicada con anterioridad en diversas revistas médicas por el equipo que lidera el Dr. J. Andonegui ${ }^{1,2}$. El Servicio de Oftalmología del Hospital Donostia aporta su experiencia al respecto en este número de la revista Anales del Sistema Sanitario de Navarra ${ }^{3}$, obtenida en 2.444 diabéticos entre diciembre de 2008 y mayo de 2010. Llama la atención la baja prevalencia observada, aunque es probable que, como dicen los autores, haya influido de forma notable el haber descartado previamente aquellos diabéticos diagnosticados de RD. Un dato importante que no se comunica es la duración de la diabetes. El hecho de que el $15,02 \%$ estuvieran sólo en tratamiento dietético y el $62,55 \%$ tomara antidiabéticos orales, hace suponer que la duración de la diabetes no era muy prolongada ya que, como demostró el UK Prospective Diabetes Study $y^{4}$ el control de la diabetes empeora con el paso de los años cualquiera que sea el tratamiento que lleve el paciente y suele requerirse, con el tiempo, el uso de insulina tras pasar por los distintos escalones terapéuticos, tal como se describe en el algoritmo más utilizado ${ }^{5}$. En consecuencia, una evolución media-corta también contribuiría a explicar la baja prevalencia de RD observada. En cualquier caso, teniendo 
en cuenta que, ya al diagnóstico, en la diabetes tipo 2 se ha descrito la presencia de RD entre el 6 y el $30 \%$ de pacientes ${ }^{6}$, sigue siendo llamativa la cifra del 9,36\% obtenida por los autores. Otros datos que se echan en falta en el artículo son: el porcentaje de diabéticos tipo 1 y tipo 2 de la muestra, el grado de control metabólico y la presencia o ausencia de nefropatía diabética e hipertensión arterial, todos ellos factores importantes a la hora de valorar los datos obtenidos.

Al hilo de esta cuestión cabe hacerse dos preguntas: en primer lugar cuándo debe observarse el fondo de ojo mediante retinógrafo no midriático y cuándo directamente por un oftalmólogo y, en segundo, con qué periodicidad debe repetirse la exploración.

Existe general acuerdo en que la visita inicial debe practicarla un oftalmólogo de forma exhaustiva, con pupila dilatada. Esta visita se realizará, en el caso de los diabéticos tipo 1, a partir de los 12 años de edad (o de la pubertad si ésta llega antes) y 5 de evolución, y en los tipo 2 cuanto antes tras el diagnóstico, ya que no sabemos cuánto tiempo pueden llevar siendo diabéticos sin ser conscientes de ello ${ }^{7-9}$. En cuanto a la frecuencia de visitas y quién debe hacerlas, hay más controversia. La ADA (Asociación de Diabetes de EEUU) considera que debe examinarse el fondo del ojo anualmente, tanto para diabéticos tipo 1 como para tipo 2, aunque si el paciente ha tenido ya una o más exploraciones

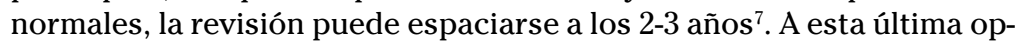
ción se apunta el Ministerio de Sanidad, Igualdad y Política Social que lo aumentaría hasta los 4 años para los diabéticos tipo 1 sin RD previa ${ }^{8}$. En Navarra, en cambio, la Guía de Actuación para Diabetes tipo 2 en Atención Primaria ${ }^{9}$ que se está revisando en la actualidad, mantiene la recomendación de la periodicidad anual. Queda claro en todos los casos que, si existe retinopatía de evolución desfavorable, las visitas serán más frecuentes.

Por otra parte, la ADA acepta que las exploraciones sean realizadas con retinógrafo (con o sin pupila dilatada) siempre y cuando sean interpretadas por personal experto y se alternen con los exámenes directos por oftalmólogo. Consideran especialmente indicado este método fotográfico cuando no hay suficientes oftalmólogos disponibles $^{7}$. El Ministerio de Sanidad, Igualdad y Política Social lo considera una alternativa adecuada en todos los casos y además concluye que la lectura pueden realizarla médicos de Atención Primaria formados previamente. En Navarra, comunidad en la que se han llevado a cabo estudios que utiliza el Ministerio de Sanidad, Igualdad y Política Social para basar sus conclusiones ${ }^{1}$, la guía indica que, tras la primera visita por oftalmólogo, la valoración posterior del fondo de ojo puede hacerse bien por oftalmólogo o bien por fotografía de retina con retinógrafo no midriático 9 .

Contemplando este tema a través del prisma de la gestión, cabe considerar su efectividad, fiabilidad y coste-eficacia. Según la British Diabetes Association y la Declaración de Saint Vincent, se considera como estándar mínimo para una prueba de cribado de la RD una sensibilidad de, al menos, $80 \%$ y una especificidad de, al menos, 95\%, con una tasa de fallo técnico de menos del 5\%. Comparado con el método habitual de cribado, que es la oftalmoscopia realizada por el oftalmólogo, la cámara de retina no midriática ofrece una sensibilidad 
mayor (81-93\%) con una especificidad similar (96\%). Ello convierte a esta técnica como una alternativa de precisión parecida a la oftalmoscopia indirecta y a la fotografía estereoscópica del fondo del ojo como método de cribado de la RD aunque no es adecuada para evaluar el edema macular 6 .

La fiabilidad comparada también con la fotografía estereoscópica y la oftalmoscopia es buena, valorado tanto como concordancia simple como con valores kappa con un nivel de confianza del 95\%. También diversos estudios llevados a cabo en España ${ }^{10}$, otros países europeos y EEUU han demostrado que el cribado con retinografía no midriática es coste-efectivo y más barato que la oftalmoscopia indirecta.

Las características del Sistema Nacional de Salud, público y gratuito, exigen soluciones imaginativas para afrontar los retos que derivan de la creciente demanda frente a una oferta lastrada por las limitaciones presupuestarias, sobre todo en tiempos de crisis económica como los actuales. La retinografía no midriática apoyada en la telemedicina es un buen exponente de estas respuestas de adaptación al medio. Sólo queda velar para que se haga con la máxima agilidad y corrección a fin de no perjudicar la detección precoz y el tratamiento adecuado de esta complicación microangiopática que acompaña con excesiva frecuencia a la diabetes.

\section{BIBLIOGRAFÍA}

1. Andonegui J, Berástegui L, Serrano L, Egúzkiza A, Gaminde I, Aliseda D. Concordancia en el estudio de retinografías en diabéticos: oftalmólogos vs médicos de familia. Arch Soc Esp Oftalmol 2008; 83: 527-532.

2. Andonegu J, Serrano L, Egúzkiza A. eOftalmología: estado actual y tendencias futuras. An Sist Sanit Navar 2010; 33: 79-91.

3. Gibelalde A, Ruiz-Miguel M, Mendicute J, Ayerdi S, Martínez-Zabalegi D. Prevalencia de retinopatía diabética mediante cribado con retinógrafo no midriático. An Sist Sanit Navar 2010; 33: 271-276.

4. UK Prospective Diabetes Study (UKPDS) Group. Intensive blood glucose control with sulphoylureas or insulin compared with conventional treatment and risk of complications in patients with type 2 diabetes (UKPDS 33) Lancet 1998; 352: 837-853.

5. Nathan DM, Buse JB, Davidson MB, Heine RJ, Holman RR, Sherwin R et al. Management of hyperglycemia in type 2 diabetes: a consensus algorithm for the initiation and adjustment of therapy. A consensus statement from the American Diabetes Association and the European Association for the study of diabetes. Diabetes Care 2006; 29: 1963-1972.

6. Vázquez San Miguel F, Goñ Iriarte MJ, Martínez Alday N. Retinografía con cámara de retina no midriática para el cribado de la retinopatía diabética. En: Picón César MJ y Ruíz de Adana M coordinadoras. Tecnologías aplicadas a la diabetes. Madrid: Edigrafos SA 2009: 103-124.

7. American Diabetes Association. Standards of Medical Care in Diabetes. Diabetes Care 2010; 33; (Suppl. 1): S11-S61.

8. García Rodríguez S, Carrasco Gimeno JM, Martín Sánchez Ji, Mengual Gil JM. Complicaciones de la diabetes. Retinopatía Diabética. Plan de Calidad para el Sistema Nacional de Salud del Ministerio de Sanidad y Política Social. Instituto Aragonés de Ciencias de la Salud; 2009. Informes de Evaluación de Tecnologías Sanitarias: I+CS № 2007/06-1. 
9. Aragón lF, Barbería JJ, Berraondo Mr, Díez J, Lafita J, Marí G et al. Diabetes Mellitus tipo 2 en Atención Primaria. Guía de actuación. Servicio Navarro de Salud-Osasunbidea. Gobierno de Navarra. Pamplona, 2006.

10. Márquez-Peláez S, Canto Neguillo R, García Mochón L, Orly de Labry lima A, BerMÚDEZ TAMAYO C. Telemedicina en el seguimiento de enfermedades crónicas: diabetes mellitus. Revisión sistemática de la literatura y evaluación económica. Plan de Calidad para el Sistema Nacional de Salud del Ministerio de Sanidad y Consumo. Agencia de evaluación de tecnologías sanitarias de Andalucía, 2008. 